\title{
Vestibular Impairment in Frontotemporal Dementia Syndrome
}

\author{
Kiyotaka Nakamagoe $^{a} \quad K^{\prime}$ otarou Kadono ${ }^{a}$ Tadachika Koganezawa $^{b}$ \\ Mao Takiguchi ${ }^{a}$ Makoto Terada ${ }^{a}$ Fumiko Yamamoto ${ }^{a}$ \\ Tetsuya Moriyama $^{a}$ Kumi Yanagiha ${ }^{a}$ Seitaro Nohara ${ }^{a}$ Naoki Tozaka ${ }^{a}$ \\ Zenshi Miyake $^{a}$ Satoshi Aizawa ${ }^{a}$ Kentaro Furushoc ${ }^{c}$ Akira Tamaoka ${ }^{a}$ \\ ${ }^{a}$ Department of Neurology, Division of Clinical Medicine, and ${ }^{b}$ Department of Physiology, \\ Division of Biomedical Science, Faculty of Medicine, University of Tsukuba, Tsukuba, and \\ 'Department of Neurology, Ryugasaki Saiseikai Hospital, Ryugasaki, Japan
}

\section{Key Words}

Frontotemporal dementia - Vestibular function - Frontotemporal lobar degeneration - Visual suppression · Vestibular stimulation · Frontal eye field · Inferior parietal lobule · Caloric test

\section{Abstract}

Background: No studies to date have attempted to evaluate frontotemporal lobar degeneration from the perspective of the vestibular system. Objective: The present study examined vestibular function in patients with frontotemporal dementia (FTD) clinical syndrome and evaluated whether vestibular disorders are involved in the clinical symptoms due to FTD. Methods: Fourteen patients with FTD syndrome, as well as healthy elderly controls without dementia, were included in the present study. All subjects underwent vestibular function tests using electronystagmography, such as caloric tests and visual suppression (VS) tests, in which the induced caloric nystagmus was suppressed by visual stimuli. The association between clinical symptoms and vestibular function in the FTD syndrome group was further examined. Results: In the FTD syndrome group, caloric nystagmus was not necessarily suppressed during VS tests. Furthermore, VS was observed to be significantly impaired in FTD syndrome patients with gait disturbance as compared to those without such disturbance. Conclusion: The present study revealed that impairment of VS in patients with FTD results in an inability to regulate vestibular function by means of visual perception, regardless of multiple presumed neuropathological backgrounds. This could also be associated with gait disturbance in patients with FTD syndrome. 


\section{Introduction}

It has long been believed that the cerebellum uses neural circuits in the vestibular system to process visual input information from the eye and then regulates the brain stem vestibular nucleus. However, several studies have contended that the cerebral cortex may be a key player in the neural circuits in the vestibular system [1-5]. Previous animal experiments have demonstrated that multiple vestibular cortex areas are scattered over the parietal and temporal lobes [6-12]. In addition, previous studies that used functional magnetic resonance imaging and positron emission tomography to investigate vestibular afferent pathways by means of vestibular stimulation have shown that the vestibular cortex area also seems to be present in the human brain $[1,2,4]$. In addition, experiments on monkeys have demonstrated not only that the vestibular cortex receives projections from the afferent fibers originating from the vestibular nuclei on both sides, but also that efferent fibers originating from the vestibular cortex directly project to the vestibular nuclei $[13,14]$. Other studies have suggested that the frontal and temporal lobe regions (including the frontal eye field, the temporal pole, the inferior temporal gyrus, and the fusiform gyrus) and the medial parietooccipital visual area may exert an inhibitory effect on the vestibular cortex via visual information $[1,2,4]$. A negative correlation is seen between activation of the visual cortical regions and activation of the vestibular cortex [1,2,4].

We previously reported that Alzheimer's disease-related pathology in the cerebral cortex can cause impairment of vestibular function which, in turn, leads to impaired visual control [5]. Taking into account the above-mentioned findings, the present study proposes a hypothesis about a system in which the cerebral cortex uses visual information to control vestibular neural circuits in Alzheimer's disease patients. We examined whether this hypothesis holds true for frontotemporal dementia (FTD) syndrome patients while no lesion was found in the cerebellum.

FTD syndrome is a clinical diagnosis and a disease characterized by symptoms that include lack of consciousness of the disease, disinhibition, character changes, dysphasia, and unsocial behavior [15-17]. FTD syndrome contrasts clinically with Alzheimer's disease; as a clinical concept, it refers to a syndrome of non-Alzheimer degeneration presenting atrophy and functional disorder mainly of the frontal and temporal lobes. Among patients with dementia, the prevalence of frontotemporal lobar degeneration ranks after that of Alzheimer's disease and Lewy body disease. Among those aged 65 years and younger, its prevalence is known to be on a par with that of Alzheimer's disease [18].

Many studies of eye movements focus on saccade disorders; these movements have been shown to be a result of frontal lobe disorders, such as frontal eye field disorder [19-22]. The frontal eye field is a region of major lesion in FTD syndrome. On the other hand, no studies to date have attempted to evaluate FTD syndrome from the perspective of the vestibular system.

Vestibular stimulation was used in patients with lesions involving mainly the cerebral cortex to examine how such stimulation may affect the visual suppression (VS) of vestibular nystagmus. This allowed us to disregard the possible effects of cerebellar deficits, thus clarifying the mechanism through which the cerebral cortex controls the vestibular neural circuits.

The aim of this study was to determine whether vestibular impairment is present in FTD clinical syndrome and, if it is present, to examine its relationship to clinical symptoms. 


\section{Subjects and Methods}

\section{Subjects}

The study subjects were 14 FTD syndrome patients ( 6 men, 8 women, age $67.9 \pm 5.7$ years). Patients in the FTD group were diagnosed in accordance with the Lund-Manchester criteria [23].

The FTD group was compared to a control group comprising 14 elderly people without dementia ( 6 men, 8 women, age $69.2 \pm 2.8$ years). Healthy voluntary subjects were recruited and given an interview and a medical examination; the control group was formed from those who scored 24 or higher on the Mini-Mental State Examination (MMSE) [24]. The control subjects were examined neurologically to confirm the absence of limb muscle weakness, extrapyramidal symptoms, deep sensibility disturbances or other sensory disorders, and cerebellar ataxia or other neurological abnormalities.

All subjects were given otolaryngological examinations. Subjects with disease of the outer or middle ear were excluded from the study, as were subjects who were taking drugs that influence vestibular function. Informed consent was obtained from all subjects in accordance with the rules of the ethics committee of the University of Tsukuba Hospital.

There was no significant difference in mean age between the FTD group and the control group ( $\mathrm{p}=0.43$, Mann-Whitney U test).

\section{Evaluation of Clinical Symptoms}

The following clinical symptoms were evaluated by neurological examination: (1) frontal lobe manifestations, (2) upward or downward gaze palsy, (3) motor neuron disorder, (4) cerebellar ataxia, (5) parkinsonism, (6) gait disturbance (10-meter gait), and (7) inability to walk (1-meter gait). We defined gait disturbance (10-meter gait) as the inability to walk continuously for $10 \mathrm{~m}$ or more unaided. We defined inability to walk as the inability to walk continuously for $1 \mathrm{~m}$ or more unaided. The tests for gait disturbance and inability to walk were carried out according to the 10-meter walking test and the 1-meter walking test, respectively.

\section{Equilibrium Function Test}

The caloric test is an effective test of vestibular function. The vestibular semicircular canals are sensitive to acceleratory stimulation due to rotation of the head, which causes a flow in the endolymph. A caloric stimulus can also cause a convective flow in the endolymph due to a difference in temperature; this stimulates the sensory hair cells, causing nystagmus. The caloric test is a vestibular stimulation test in which a flow in the endolymph is purposely provoked by irrigating air or water at a temperature different from that of the body into the external auditory canal. This induces nystagmus, allowing the reaction of the vestibular system's neural pathways to be seen. Nystagmus induced in this way is called caloric nystagmus, while the suppression of caloric nystagmus due to visual fixation is called visual suppression. VS impairment is known to be caused by disorders not only of the cerebellum and brainstem, but also of the cerebral cortex [25-27]. The level of VS is measured as an equilibrium function test, which is helpful in diagnosing central nervous system disorders.

Eye movement was recorded by attaching electrodes in horizontal and vertical directions to the eyelid. The original waveform (time constant $=3 \mathrm{~s}$ ) and velocity waveform (time constant $=0.03 \mathrm{~s}$ ) of eye movements in each direction were recorded using an electronystagmograph and a data collection and analysis device with a sampling frequency of $1 \mathrm{kHz}$ (PowerLab; ADInstruments Pty. Ltd., Castle Hill, Australia).

The caloric test was carried out with the subject in the supine position with the head raised $30^{\circ}$ and wearing goggles fitted with an infrared camera (First, Tokyo, Japan). During 
the test, the open or shut status of the eyes was monitored visually, and the electronystagmogram was recorded. The caloric stimulus to induce nystagmus was delivered by irrigating with cool air at $24^{\circ} \mathrm{C}$ for $1 \mathrm{~min}$ from the external auditory canal to the eardrum, using an air caloric device (First). Forty seconds after the end of the stimulus, the VS test was carried out with visual fixation by gazing at a target for $10 \mathrm{~s}$. The nystagmus induced was analyzed, and the parameters of frequency, amplitude (slow and quick phase), and mean velocity (slow and quick phase) were investigated. Mean values of each parameter were calculated for the $10 \mathrm{~s}$ before the VS test and the $10 \mathrm{~s}$ during the VS test; these were separated into before the VS test and during the VS test for analysis.

The suppression rate (SR) of slow-phase velocity during the VS test was expressed as a percentage using the following equation: $\mathrm{SR}=100 \times(\mathrm{VbVS}-\mathrm{VdVS}) /(\mathrm{VbVS})$, where VbVS is the slow-phase velocity before the VS test and VdVS is the slow-phase velocity during the VS test. The SR for each subject was calculated using this equation; the mean SR value for each group was subsequently calculated.

The velocity of the slow phase is normally lower during the VS test than before the VS test. The SR is usually a positive value; however, where the slow-phase velocity was greater during the VS test than before the VS test, regarding an increase in caloric nystagmus, the SR value was negative.

Evaluation of Brain Lesions Using Cerebral Blood Flow Single-Photon Emission Computed Tomography Tests

${ }^{123}$ I-IMP single-photon emission computed tomography (SPECT) brain imaging was carried out to evaluate cerebral function as a diagnostic aid in the FTD group. Tomographic statistical analysis (issp5/isspTomo; Nihon Medi-Physics Co., Ltd., Tokyo, Japan) was performed on the SPECT images, and Z score images were evaluated by comparing them with the normal database of the University of Tsukuba [28]. The database of the University of Tsukuba comprises 23 cases aged $66-79$ years. A Z score $\geq 2.0$ was judged to be a significant difference.

A statistical image analysis of the cerebral blood flow SPECT was used to investigate the reduction in blood flow in the frontotemporal lobes, but not the cerebellum, in the FTD syndrome group.

\section{Statistical Analysis}

The data obtained were tested statistically with the Mann-Whitney U test, using statistics software (IBM SPSS Statistics base software; IBM, New York, N.Y., USA). The level for a statistically significant difference was $5 \%$.

\section{Results}

\section{Clinical Symptoms of Subjects with FTD Syndrome}

The clinical characteristics of the FTD syndrome patients are compiled in table 1 . The mean age at onset was $64.8 \pm 7.1$ years, and the mean disease duration was $3.4 \pm 2.9$ years. In the FTD group, the mean MMSE score was $21.9 \pm 5.0$, and the mean Frontal Assessment Battery [29] score was $8.8 \pm 3.6$. MMSE and Frontal Assessment Battery scores were both significantly lower in the FTD group than in the healthy control group $(\mathrm{p}<0.05$, MannWhitney U test) (table 1).

The most common symptom was character change, which was present in all 14 cases, while perseveration was found in 10 cases, non-fluent aphasia in 2 cases, vertical gaze palsy in 8 cases, upper motor neuron disorder in 4 cases, lower motor neuron disorder in 5 cases, and parkinsonism in 10 cases. 
Table 1. Clinical characteristics of the two groups

\begin{tabular}{lccl}
\hline & $\begin{array}{c}\text { FTD syndrome } \\
(\mathrm{n}=14)\end{array}$ & $\begin{array}{c}\text { Control } \\
(\mathrm{n}=14)\end{array}$ & p value \\
\hline Age, years & $67.9 \pm 5.7$ & $69.2 \pm 2.8$ & 0.434 \\
Males/females & $6 / 8$ & $6 / 8$ & - \\
MMSE & $21.9 \pm 5.0$ & $28.6 \pm 1.2$ & $0.0000007^{* *}$ \\
Frontal Assessment Battery & $8.8 \pm 3.6$ & $15.9 \pm 1.3$ & $0.0000007^{* *}$ \\
Age at onset, years & $64.8 \pm 7.1$ & - & - \\
Years affected & $3.4 \pm 2.9$ & - & - \\
Clinical symptoms & & & - \\
$\quad$ Perseveration & $10(71.4)$ & - & - \\
$\quad$ Non-fluent aphasia & $2(14.3)$ & - & - \\
Vertical gaze palsy & $8(57.1)$ & - & - \\
$\quad$ Upper motor neuron disorder & $4(33.3)$ & - & - \\
$\quad$ Lower motor neuron disorder & $5(35.7)$ & - & - \\
$\quad$ Cerebellar ataxia & $0(0)$ & - & - \\
$\quad$ Parkinsonism & $10(71.4)$ & - & - \\
Gait disturbance (10-meter gait) & $3(21.4)$ & - & - \\
$\quad$ Inability to walk (1-meter gait) & $6(42.9)$ & - & - \\
Decreased blood flow in the frontotemporal lobe & $14(100.0)$ & - & - \\
Decreased blood flow in the inferior parietal lobule & $4(28.6)$ & - & - \\
Decreased blood flow in the cerebellum & $0(0)$ & - & - \\
\hline
\end{tabular}

Figures are presented as means \pm standard deviations, $\mathrm{n} / \mathrm{n}$ or $\mathrm{n}(\%)$.

** Intergroup comparison with the control group ( $p<0.01$, Mann-Whitney U test).

a
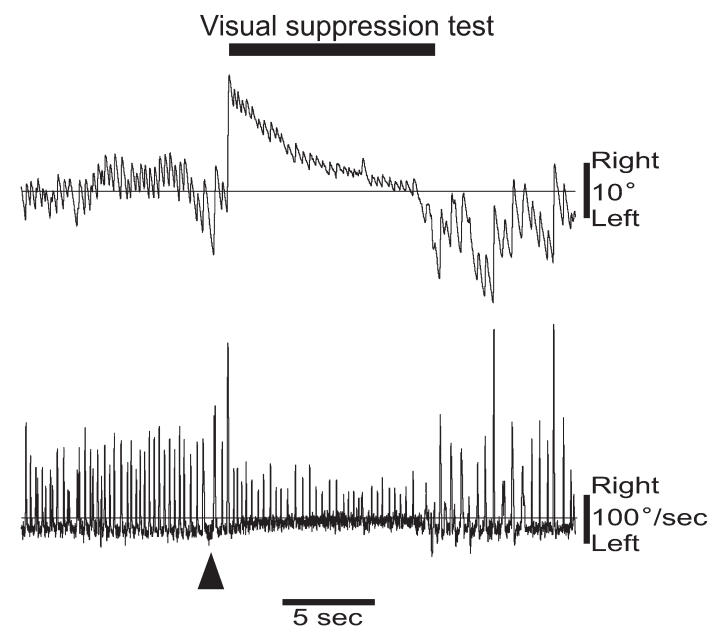

b
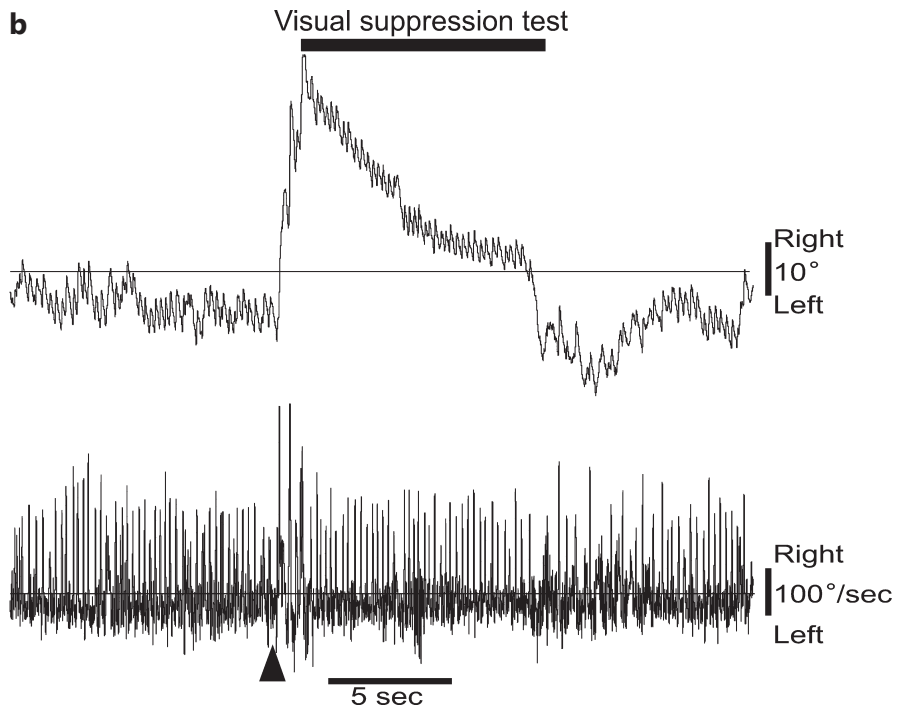

Fig. 1. Examples of caloric tests. Results of stimulation of the left ear with cool air at $24^{\circ} \mathrm{C}$ are presented. The upper panel shows horizontal eye movement, the lower panel shows velocity. Arrowheads indicate calculated SR values of 78\% (a; a healthy 74-year-old male subject) and 38\% (b; a 68-year-old FTD syndrome subject). 
Table 2. Caloric nystagmus test

\begin{tabular}{|c|c|c|c|}
\hline & $\begin{array}{l}\text { FTD syndrome } \\
\text { ( } 28 \text { ears) }\end{array}$ & $\begin{array}{l}\text { Control } \\
\text { (28 ears) }\end{array}$ & $\mathrm{p}$ value \\
\hline \multicolumn{4}{|l|}{ Pre-VS test } \\
\hline \multicolumn{4}{|l|}{ Amplitude, degrees } \\
\hline Quick phase & $3.1 \pm 1.7$ & $5.5 \pm 1.6$ & $0.000002^{* *}$ \\
\hline Slow phase & $3.3 \pm 1.8$ & $5.6 \pm 1.7$ & $0.00001^{* *}$ \\
\hline \multicolumn{4}{|l|}{ Velocity, degrees/s } \\
\hline Quick phase & $59.4 \pm 21.5$ & $82.1 \pm 17.3$ & $0.00008^{* *}$ \\
\hline Slow phase & $12.4 \pm 7.2$ & $15.2 \pm 3.7$ & 0.076 \\
\hline Nystagmus frequency, /s & $3.0 \pm 1.1$ & $2.5 \pm 0.7$ & 0.063 \\
\hline \multicolumn{4}{|l|}{ VS test } \\
\hline \multicolumn{4}{|l|}{ Amplitude, degrees } \\
\hline Quick phase & $3.2 \pm 1.8$ & $1.9 \pm 0.6$ & $0.0004^{* *}$ \\
\hline Slow phase & $4.0 \pm 2.0$ & $2.3 \pm 0.8$ & $0.0002^{* *}$ \\
\hline \multicolumn{4}{|l|}{ Velocity, degrees/s } \\
\hline Quick phase & $65.5 \pm 24.9$ & $45.6 \pm 9.0$ & $0.0002^{* *}$ \\
\hline Slow phase & $11.4 \pm 8.9$ & $5.7 \pm 2.6$ & $0.002^{* *}$ \\
\hline Nystagmus frequency, /s & $2.4 \pm 1.3$ & $2.1 \pm 0.9$ & 0.310 \\
\hline $\mathrm{SR}, \%$ & $4.8 \pm 46.0$ & $63.1 \pm 12.2$ & $0.00000003^{* *}$ \\
\hline
\end{tabular}

Figures are presented as means \pm standard deviations.

** $\mathrm{p}<0.01$ as compared to healthy controls; Mann-Whitney U test.

FTD may be broadly classified into three subtypes: behavioral variant FTD, progressive non-fluent aphasia, and semantic dementia $[15,16]$. There are also non-typical cases that do not correspond to any of these clinical forms. In the present study, there were 12 cases of behavioral variant FTD and 2 cases of progressive non-fluent aphasia; none of the subjects had semantic dementia.

\section{Caloric Test Results for Each Group}

Caloric tests were carried out on 14 people (28 ears) in the FTD group and on 14 people (28 ears) in the control group (fig. 1). Before the VS test, quick-phase amplitude, slow-phase amplitude, and quick-phase velocity were significantly lower in the FTD group than in the control group. During the VS test, both the amplitude and the velocity of the quick and slow phases were significantly higher in the FTD group than in the control group (table 2). The mean SR value was significantly lower in the FTD group than in the control group. Additionally, findings of increased nystagmus were present during the VS test in more than half $(57.1 \%)$ of the subjects.

\section{Relationship of Subjective Symptoms with SR in the FTD Group}

The mean SR values of patients in the FTD group with different clinical symptoms were compared (table 3). The SR value was significantly lower in patients with perseveration, patients with gait disturbance, and patients unable to walk. The SR showed no relationship with non-fluent aphasia, vertical gaze palsy, upper motor neuron disorder, lower motor neuron disorder, or parkinsonism. 
Table 3. Relationship of the SR to neurological signs in the FTD group ( $n=14)$

\begin{tabular}{llcl}
\hline \multirow{2}{*}{ Clinical symptoms } & \multicolumn{2}{l}{ SR, $\%$} & \multirow{2}{*}{ p value } \\
\cline { 2 - 3 } & $\begin{array}{l}\text { FTD syndrome with } \\
\text { neurological signs }\end{array}$ & $\begin{array}{l}\text { FTD syndrome without } \\
\text { neurological signs }\end{array}$ & \\
\hline Perseveration & $17.4 \pm 32.0(10 / 14)$ & $-22.3 \pm 22.2(4 / 14)$ & $0.029^{*}$ \\
Non-fluent aphasia & $-8.4 \pm 23.3(2 / 14)$ & $8.4 \pm 35.9(12 / 14)$ & 0.482 \\
Vertical gaze palsy & $2.2 \pm 29.2(8 / 14)$ & $11.2 \pm 42.4(6 / 14)$ & 0.667 \\
Upper motor neuron disorder & $10.1 \pm 38.7(4 / 14)$ & $4.4 \pm 34.4(10 / 14)$ & 0.809 \\
Lower motor neuron disorder & $-5.1 \pm 36.1(5 / 14)$ & $12.2 \pm 33.6(9 / 14)$ & 0.406 \\
Parkinsonism & $14.8 \pm 28.5(10 / 14)$ & $-16.0 \pm 41.7(4 / 14)$ & 0.244 \\
Gait disturbance (10-meter gait) & $-41.0 \pm 10.6(3 / 14)$ & $18.9 \pm 25.6(11 / 14)$ & $0.0002^{* *}$ \\
Inability to walk (1-meter gait) & $-15.3 \pm 32.8(6 / 14)$ & $22.0 \pm 26.9(8 / 14)$ & $0.048^{*}$ \\
\hline
\end{tabular}

Figures are presented as means \pm standard deviations, with number of patients in parentheses.

* Intergroup comparison based on the presence or absence of signs and symptoms $(\mathrm{p}<0.05$, MannWhitney U test). ${ }^{* *}$ Intergroup comparison based on the presence or absence of signs and symptoms $(\mathrm{p}<0.01$, Mann-Whitney U test).

\section{Discussion}

Although FTD syndrome has various clinical symptoms with multiple presumed neuropathological backgrounds, a large number of cases with FTD syndrome had impaired ability to suppress caloric nystagmus by visual fixation. Furthermore, VS impairment showed a significant relationship to the inability to walk in FTD syndrome. Based on the previous findings as outlined above, the mechanism through which VS impairment appears in cortical dysfunction was speculated to be as presented in figure 2 [1, 2, 4, 5, 30, 31].

Figure 2a shows hypothesized vestibulo-ocular reflex neural pathways after stimulation of the horizontal semicircular canal in healthy individuals. Stimulation of the horizontal semicircular canal by cold air causes the inhibitory neurons in the vestibular nucleus to decrease their inhibition of activity in the abducens nucleus (IV). On the other hand, the activity of the oculomotor nucleus (III) ipsilateral to the vestibular-stimulated side does not change. As a result, only the tonus of the abductor muscles increases, which causes them to contract, thus producing the slow phase of caloric nystagmus.

Figure $2 \mathrm{~b}$ shows the hypothesized route of VS in healthy individuals. During a VS test, vision-related regions, including the cerebellum and the frontal eye field in the cerebral cortex, receive visual stimulation. Floccular Purkinje cells contralateral to the vestibularstimulated side are activated by visual stimulation, exerting an increased inhibitory effect on the inhibitory neurons in the vestibular nucleus. As a result, inhibitory neuronal activity in the vestibular nucleus becomes disrupted, exerting less of an inhibitory effect on the excitatory neurons in the abducens nucleus contralateral to the vestibular-stimulated side (IV). Therefore, the excitatory neurons in the abducens nucleus contralateral to the vestibularstimulated side (IV) become activated, exerting a greater excitatory effect on the extraocular motor nuclei on the vestibular-stimulated side (III), which increases the tonus of the adductor muscles on the vestibular-stimulated side. Based on previous clinical findings for lesions on the inferior parietal lobule [5,26], it can be assumed that visual stimulation might cause the vestibular cortex to exert an excitatory effect on the inhibitory neurons in the vestibular nucleus ipsilateral to the vestibular-stimulated side. The inhibitory neurons in the vestibular nucleus are therefore activated, exerting a greater inhibitory effect on the extraocular motor 
Dementia

a

Ipsilateral direction

4

r

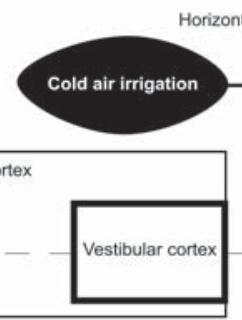

Horizontal semicircular canal

Extraocular motor neurons (IV)

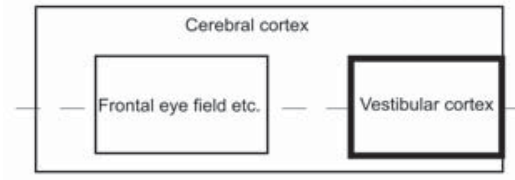

1

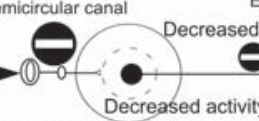

Medial vestibular nucleus

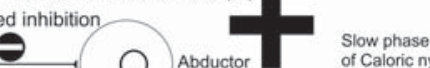

Contralateral direction

Medial vestibular nucleus Extraocular motor neurons (IV)

b

b

4

Extraocular motor neurons (III)

Middle zone of flocculus
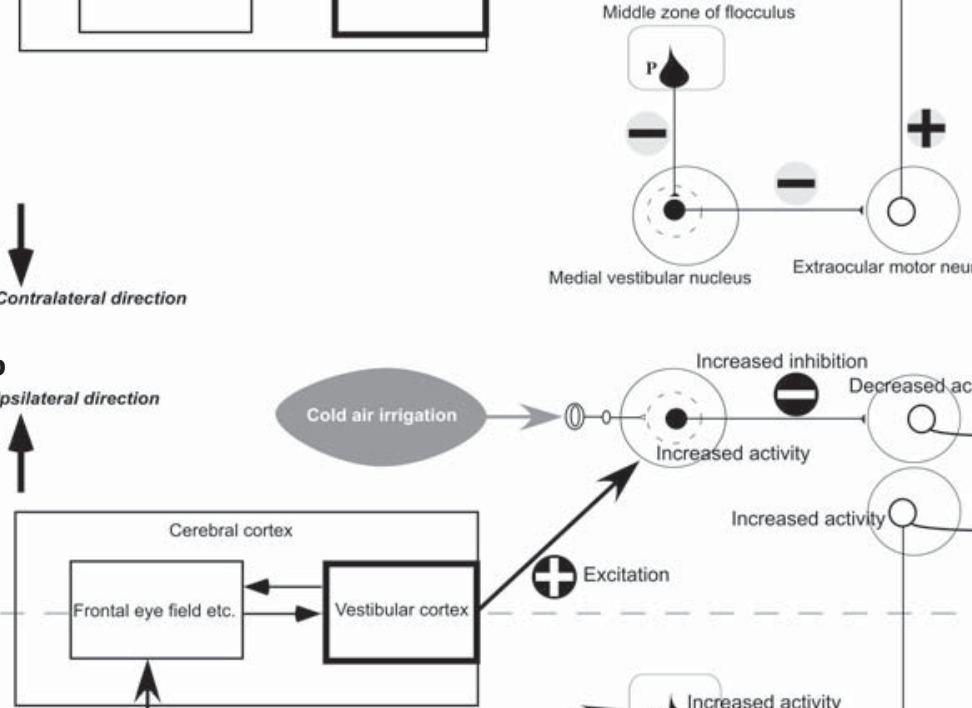

$\oplus$

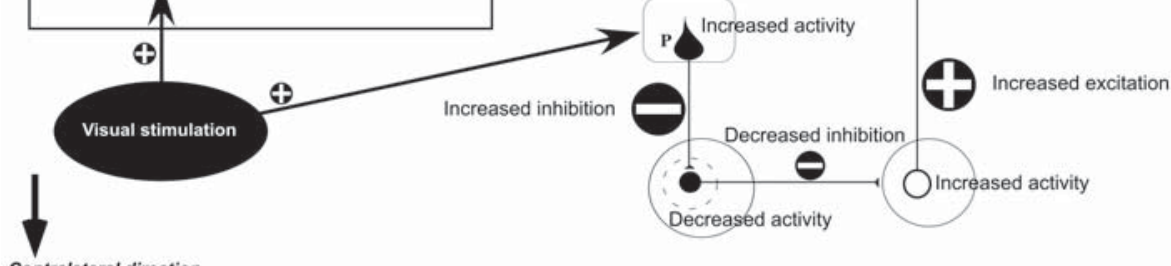

Contralateral direction

Ipsilateral direction

1
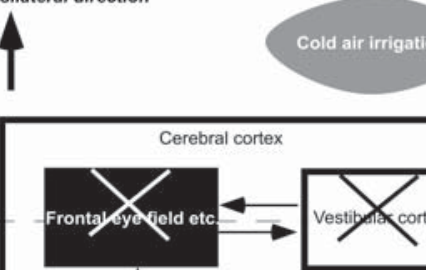

$\boldsymbol{P}$

Increased inhibition

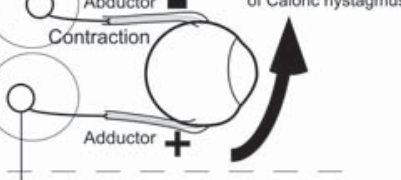

increased activity

tion

Increased activity
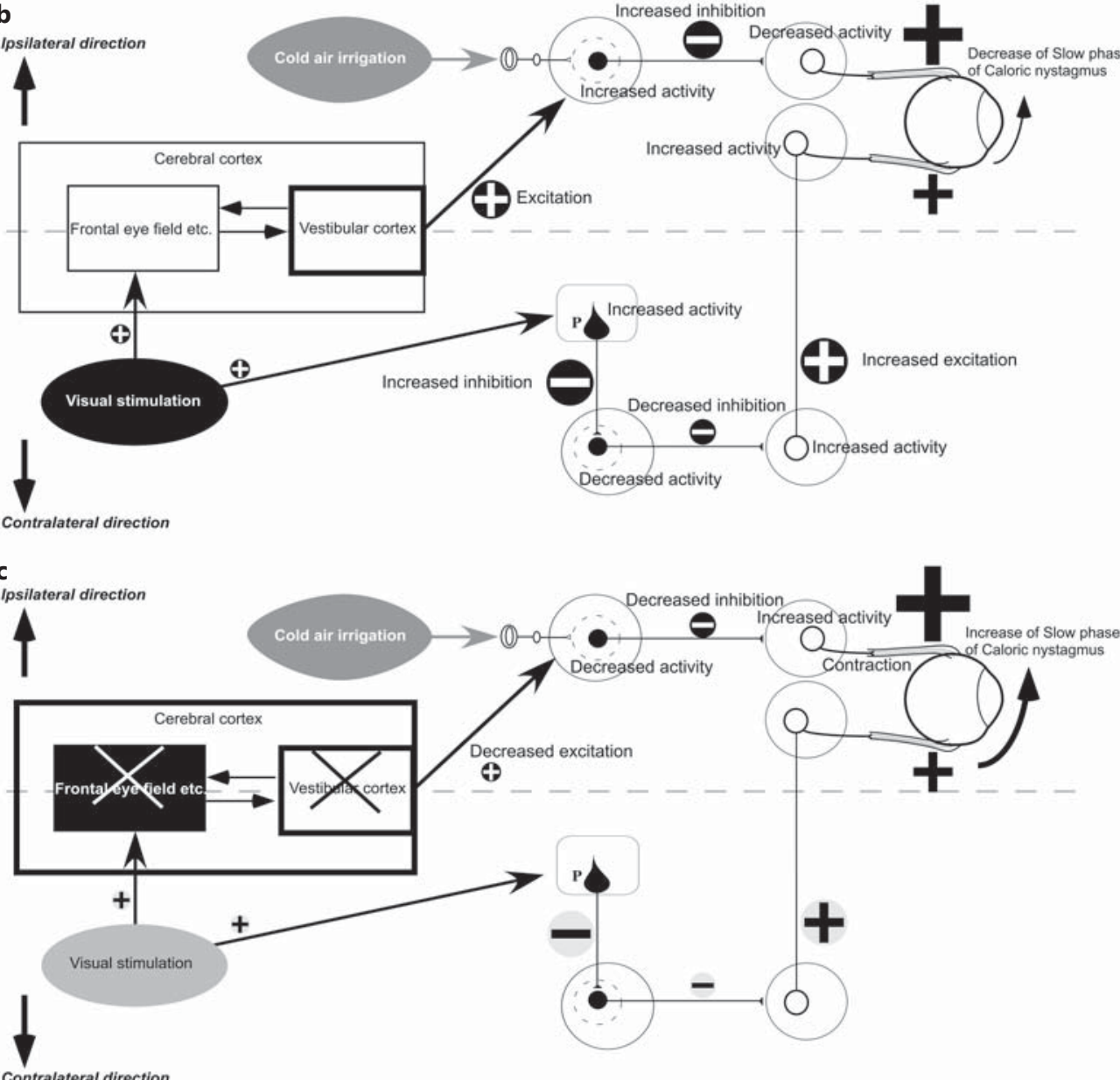

Contralateral direction

Fig. 2. Hypothesized mechanism of the disorder of VS in FTD syndrome. a Vestibulo-ocular reflex neural pathways after stimulation of the horizontal semicircular canal in healthy individuals. b Hypothesized route of VS in healthy individuals. c Hypothesized mechanism of VS disorder in FTD syndrome. III = Oculomotor nucleus; IV = abducens nucleus; $\mathrm{P}$ = Purkinje cell. White circles show excitatory neurons, black circles show inhibitory neurons. Plus signs stand for excitation, minus signs for inhibition. The size of plus and minus signs reflects the degree of activity. 
nuclei ipsilateral to the vestibular-stimulated side (IV) and potentially decreasing the tonus of the abductor muscles on the stimulated side. In view of the information cited above, when compared with figure $2 \mathrm{a}$, it is evident that the decreased tonus of the abductor muscles derived from regulatory functions performed by the cerebrum and the decreased tonus of the adductor muscles derived from regulatory functions performed by the cerebellum lead to a reduced difference between the tonus of the abductor and adductor muscles. This, in turn, potentially decreases the slow-phase velocity of caloric nystagmus, thus inhibiting vestibular nystagmus. This phenomenon is called visual suppression.

Based on previous clinical findings $[5,26]$, we speculate that damage to both the visual cortical regions and vestibular cortex might induce VS impairment of caloric nystagmus in FTD patients. FTD patients could have not only frontotemporal lesions but also parietal lesions, such as in the inferior parietal lobule [32-34]. About 30\% of FTD patients had decreased blood flow in the inferior parietal lobule in the present study. There is no report that only frontal lobe lesions induce impaired ability to suppress caloric nystagmus by visual fixation. On the other hand, frontal lobe lesions combined with parietal lobe lesions, such as in the inferior parietal lobule, could induce VS impairment [26].

Figure 2c shows the hypothesized mechanism of VS impairment in FTD syndrome. In FTD syndrome patients, degeneration affects not only visual cortical regions, such as the frontal eye field, but also the vestibular cortex, such as the inferior parietal lobule. As a result, the inhibitory neurons in the vestibular nucleus ipsilateral to the vestibular-stimulated side receive less excitatory input from the vestibular cortex. Based on previous clinical findings $[5,26]$, we speculate that the interaction between the visual cortical regions and the vestibular cortex is important for the control of the vestibular nucleus in the brainstem. When comparing with figure $2 \mathrm{~b}$, it is evident that the inhibitory neurons in the vestibular nucleus inhibit the extraocular motor nuclei less ipsilaterally to the vestibular-stimulated side (IV). This, in turn, activates the neurons in the extraocular motor nuclei (IV), which leads to increased tonus of the abductor muscles on the vestibular-stimulated side. As a consequence, the difference in muscle tonus between the abductor and adductor increases, causing the slow-phase velocity of caloric nystagmus to increase during a VS test. Inhibition of the slow phase is therefore disrupted, leading to the onset of impaired VS.

The slow phase of caloric nystagmus is eye movement that occurs as a result of the vestibulo-ocular reflex. If the activity of the vestibular semicircular canals on one side caused by caloric stimulus of the labyrinth is greater than the activity on the opposite side, there is a slow eye movement toward the opposite side; this corresponds to the slow phase of caloric nystagmus. The shortest pathway of the vestibulo-ocular reflex is a three-neuron reflex arc comprising the vestibular nerve, vestibular nucleus neurons, and extraocular motor neurons. As no disorder of slow-phase velocity was found in the FTD group in this study, it may be surmised that the function of the reflex arc of the vestibulo-ocular reflex is relatively preserved in FTD syndrome.

Based on previous reports [5, 25-27], SR and slow-phase velocity are thought to be important caloric vestibular parameters. On the other hand, we think that the other parameters, such as velocity of quick phase and amplitude, are not important in the present study, which attempted to evaluate vestibular impairment due to cortical dysfunction.

The limitations of the present study are as follows. First, the number of cases studied was extremely small. Second, it is difficult to judge the effects of vestibular disorder on gait disturbance in FTD syndrome. Factors other than vestibular disorder, such as muscle weakness and parkinsonism, have complex effects and can easily cause bias. Third, we were unable to use a neuropathological approach to FTD syndrome, such as FTLD-tau, FTLD-TDP43 (TAR DNAbinding protein of $43 \mathrm{kDa}$ ), and FUS (fusion in sarcoma/translated in liposarcoma) [35]. To clarify the clinical significance and mechanisms of vestibular disorder in FTD syndrome, future studies are needed with larger sample sizes that, as far as possible, can be examined without bias. 


\section{Conclusion}

The findings of our present study are consistent with the hypothesis that the cerebral cortex uses visual perception to regulate vestibular function through efferent pathways. The regulation of vestibular neural circuits by means of visual input was observed to be impaired in patients with FTD syndrome. The resultant vestibular balance disorder may, thus, be one factor causing gait disorders. A vestibular balance disorder in patients with FTD syndrome is a serious problem that can lead to decreased quality of life. Elucidating the series of pathways originating from the cerebral cortex that are involved in the regulation of vestibular function can, therefore, provide useful insights into the clinical management of patients with FTD syndrome.

\section{Acknowledgments}

This study was funded by a Scientific Research (C) grant (No. 26460901) from the Ministry of Education, Culture, Sports, Science and Technology of Japan.

\section{Disclosure Statement}

The authors declare no competing financial interests.

\section{References}

1 Friberg L, Olsen TS, Roland PE, Paulson OB, Lassen NA: Focal increase of blood flow in the cerebral cortex of man during vestibular stimulation. Brain 1985;108:609-623.

-2 Brandt T, Bartenstein P, Janek A, Dieterich M: Reciprocal inhibitory visual-vestibular interaction: visual motion stimulation deactivates the parieto-insular vestibular cortex. Brain 1998;121:1749-1758.

3 Guldin WO, Grüsser OJ: Is there a vestibular cortex? Trends Neurosci 1998;21:254-259.

4 Naito Y, Tateya I, Hirano S, Inoue M, Funabiki K, Toyoda H, Ueno M, Ishizu K, Nagahama Y, Fukuyama H, Ito J: Cortical correlates of vestibulo-ocular reflex modulation: a PET study. Brain 2003;126:1562-1578.

5 Nakamagoe K, Fujimiya S, Koganezawa T, Kadono K, Shimizu K, Fujizuka N, Takiguchi S, Ueno T, Monzen T, Tamaoka A: Vestibular function impairment in Alzheimer's disease. J Alzheimers Dis 2015;47:185-196.

6 Fredrickson JM, Scheid P, Figge U, Kornhuber HH: Vestibular nerve projection to the cerebral cortex of the rhesus monkey. Exp Brain Res 1966;2:318-327.

7 Schwarz DW, Fredrickson JM: Rhesus monkey vestibular cortex: a bimodal primary projection field. Science 1971;172:280-281.

8 Büttner U, Buettner UW: Parietal cortex (2v) neuronal activity in the alert monkey during natural vestibular and optokinetic stimulation. Brain Res 1978;153:392-397.

-9 Odkvist LM, Schwarz DW, Fredrickson JM, Hassler R: Projection of the vestibular nerve to the area 3a arm field in the squirrel monkey (Saimiri sciureus). Exp Brain Res 1974;21:97-105.

$\$ 10$ Faugier-Grimaud S, Ventre J: Anatomic connections of inferior parietal cortex (area 7) with subcortical structures related to vestibulo-ocular function in a monkey (Macaca fascicularis). J Comp Neurol 1989;280:1-14.

11 Grüsser OJ, Pause M, Schreiter U: Localization and responses of neurones in the parieto-insular vestibular cortex of awake monkeys (Macaca fascicularis). J Physiol 1990;430:537-557.

12 Grüsser OJ, Pause M, Schreiter U: Vestibular neurones in the parieto-insular cortex of monkeys (Macaca fascicularis): visual and neck receptor responses. J Physiol 1990;430:559-583.

13 Guldin WO, Mirring S, Grüsser OJ: Connections from the neocortex to the vestibular brain stem nuclei in the common marmoset. Neuroreport 1993;5:113-116.

14 Akbarian S, Grüsser OJ, Guldin WO: Corticofugal connections between the cerebral cortex and brainstem vestibular nuclei in the macaque monkey. J Comp Neurol 1994;339:421-437.

15 Neary D, Snowden JS, Gustafson L, Passant U, Stuss D, Black S, Freedman M, Kertesz A, Robert PH, Albert M, Boone K, Miller BL, Cummings J, Benson DF: Frontotemporal lobar degeneration: a consensus on clinical diagnostic criteria. Neurology 1998;51:1546-1554. 
16 McKhann GM, Albert MS, Grossman M, Miller B, Dickson D, Trojanowski JQ: Clinical and pathological diagnosis of frontotemporal dementia: report of the Work Group on Frontotemporal Dementia and Pick's Disease. Arch Neurol 2001;58:1803-1809.

17 Grossman M: Frontotemporal dementia: a review. J Int Neuropsychol Soc 2002;8:566-583.

18 Bian H, Grossman M: Frontotemporal lobar degeneration: recent progress in antemortem diagnosis. Acta Neuropathol 2007;114:23-29.

19 Meyniel C, Rivaud-Pechoux S, Damier P, Gaymard B: Saccade impairments in patients with fronto-temporal dementia. J Neurol Neurosurg Psychiatry 2005;76:1581-1584.

-20 Boxer AL, Garbutt S, Rankin KP, Hellmuth J, Neuhaus J, Miller BL, Lisberger SG: Medial versus lateral frontal lobe contributions to voluntary saccade control as revealed by the study of patients with frontal lobe degeneration. J Neurosci 2006;26:6354-6363.

-21 Garbutt S, Matlin A, Hellmuth J, Schenk AK, Johnson JK, Rosen H, Dean D, Kramer J, Neuhaus J, Miller BL, Lisberger SG, Boxer AL: Oculomotor function in frontotemporal lobar degeneration, related disorders and Alzheimer's disease. Brain 2008;131:1268-1281.

22 Burrell JR, Hornberger M, Carpenter RH, Kiernan MC, Hodges JR: Saccadic abnormalities in frontotemporal dementia. Neurology 2012;78:1816-1823.

23 Miller BL, Ikonte C, Ponton M, Levy M, Boone K, Darby A, Berman N, Mena I, Cummings JL: A study of the LundManchester research criteria for frontotemporal dementia: clinical and single-photon emission CT correlations. Neurology 1997;48:937-942.

24 Cockrell JR, Folstein MF: Mini-Mental State Examination (MMSE). Psychopharmacol Bull 1988;24:689-692.

25 Kato I, Sato Y, Aoyagi M, Mizukoshi K, Kimura Y, Koike Y, Hayano N: Caloric pattern test with special reference to failure of fixation-suppression. Acta Otolaryngol 1979;88:97-104.

26 Takemori S, Ono M, Maeda T: Cerebral contribution to the visual suppression of vestibular nystagmus. Arch Otolaryngol 1979;105:579-581.

27 Takemori S, Aiba T, Shizawa R: Visual suppression of caloric nystagmus in brain-stem lesions. Ann NY Acad Sci 1981;374:846-854.

28 Minoshima S, Frey KA, Koeppe RA, Foster NL, Kuhl DE: A diagnostic approach in Alzheimer's disease using three-dimensional stereotactic surface projections of fluorine-18-FDG PET. J Nucl Med 1995;36:1238-1248.

29 Dubois B, Slachevsky A, Litvan I, Pillon B: The FAB: a Frontal Assessment Battery at bedside. Neurology 2000; 55:1621-1626.

-30 Sato Y, Kawasaki T: Identification of the Purkinje cell/climbing fiber zone and its target neurons responsible for eye-movement control by the cerebellar flocculus. Brain Res Brain Res Rev 1991;16:39-64.

31 Fushiki H, Sato Y, Miura A, Kawasaki T: Climbing fiber responses of Purkinje cells to retinal image movement in cat cerebellar flocculus. J Neurophysiol 1994;71:1336-1350.

-32 Jeong Y, Cho SS, Park JM, Kang SJ, Lee JS, Kang E, Na DL, Kim SE: 18F-FDG PET findings in frontotemporal dementia: an SPM analysis of 29 patients. J Nucl Med 2005;46:233-239.

33 Seelaar H, Papma JM, Garraux G, de Koning I, Reijs AE, Valkema R, Rozemuller AJ, Salmon E, van Swieten JC: Brain perfusion patterns in familial frontotemporal lobar degeneration. Neurology 2011;77:384-392.

-34 Verfaillie SC, Adriaanse SM, Binnewijzend MA, Benedictus MR, Ossenkoppele R, Wattjes MP, Pijnenburg YA, van der Flier WM, Lammertsma AA, Kuijer JP, Boellaard R, Scheltens P, van Berckel BN, Barkhof F: Cerebral perfusion and glucose metabolism in Alzheimer's disease and frontotemporal dementia: two sides of the same coin? Eur Radiol 2015;25:3050-3059.

-35 Rademakers R, Neumann M, Mackenzie IR: Advances in understanding the molecular basis of frontotemporal dementia. Nat Rev Neurol 2012;8:423-434. 